\title{
Strong Completeness for Markovian Logics
}

\author{
Dexter Kozen ${ }^{1}$, Radu Mardare ${ }^{2}$, and Prakash Panangaden ${ }^{3}$ \\ 1. Computer Science Department, Cornell University, Ithaca, New York, USA \\ 2. Department of Computer Science, Aalborg University, Denmark \\ 3. School of Computer Science, McGill University, Montreal, Quebec, Canada
}

\begin{abstract}
In this paper we present Hilbert-style axiomatizations for three logics for reasoning about continuous-space Markov processes (MPs): (i) a logic for MPs defined for probability distributions on measurable state spaces, (ii) a logic for MPs defined for sub-probability distributions and (iii) a logic defined for arbitrary distributions. These logics are not compact so one needs infinitary rules in order to obtain strong completeness results.

We propose a new infinitary rule that replaces the so-called Countable Additivity Rule (CAR) currently used in the literature to address the problem of proving strong completeness for these and similar logics. Unlike the CAR, our rule has a countable set of instances; consequently it allows us to apply the RasiowaSikorski lemma for establishing strong completeness. Our proof method is novel and it can be used for other logics as well.
\end{abstract}

\section{Introduction}

Markov processes (MPs) are standard models used for abstracting and reasoning about complex natural and man-made systems in order to handle either a lack of knowledge or inherent randomness. There are various levels of abstraction that one can consider in the definition of Markov processes: (i) the state space can be modeled by using particular types of structures that can vary from discrete finite spaces to topological or measurable spaces; (ii) the indeterminacy can be modeled by using probability or sub-probability distributions over the state space to describe the probability of transitions or by assuming exponentially distributed random variables to characterize the time durations between transitions.

To specify properties of Markov processes, the natural logic is a simple modal logic in which bounds on probabilities enter into the modalities. This logic can be stripped down to a very spartan core - just the modalities and finite conjunction - and still characterize bisimulation for labeled Markov processes [5,6]. It is therefore tempting to understand this logic from a proof theoretic perspective. Recent papers $[4,11,19]$ have established complete proof systems and prove finite model properties for similar logics. Goldblatt in [11] presents a proof-theoretic analysis of the logic of $T$-coalgebras, where $T$ is any polynomial functor constructed from a standard monad on the category of measurable spaces. He proves that the semantic consequence relation over $T$-coalgebras is equal to the least deducibility relation that satisfies Lindenbaum's lemma, which states that any consistent set of formulas can be extended to a maximally consistent set. In other words, this consequence relation is equal to the least of all deducibility relations 
if and only if that least deducibility relation satisfies Lindenbaum's lemma. These logics are not compact and for proving the aforementioned results in [11] it is used a powerful infinitary axiom scheme named the Countable Additivity Rule (CAR). In [21] Zhou and Ying prove that such a logic is not strongly-complete in the absence of CAR.

A feature of CAR is that it has an uncountable set of instances. This fact makes it difficult to prove that maximally consistent sets exist for such logics and consequently, in the papers concerned with the strong completeness of the modal logics for Harsanyi type spaces $[19,21]$ or for Markov processes $[4,15]$ it had to be assumed that consistent sets can be extended to maximally consistent sets. The completeness theorems cited are contingent on this assumption.

In this paper we reconsider the axiomatizations of the deducibility relations for three modal logics for Markov processes. The first one refers to what we call probabilistic Markov processes (PMPs), which are Markov processes defined by probability distribution over the state space. The second one is a modal logic for subprobabilistic Markov processes (SMPs), which are Markov processes defined for sub-probability distributions, and the third one is defined for what we call general Markov processes (GMPs), which are Markov processes defined for arbitrary distributions, usually these are interpreted as rates.

We propose a new infinitary axiom schema to replace CAR. Unlike CAR, our axiom has a countable set of instances. This fact allows us to invoke the Rasiowa-Sikorski Lemma and prove the strong completeness theorem via a canonical models construction without needing to assume that consistent sets can be enlarged to maximal consistent sets (Lindenbaum's lemma). In fact Lindenbaum's lemma can be directly proven from the Rasiowa-Sikorski lemma.

The Rasiowa-Sikorski lemma is a model-theoretic result that exploits a topological result known as Baire category theorem and the Stone duality for boolean algebras with operators. Applied to logics, the Rasiowa-Sikorski lemma states that given a multimodal logic (possibly involving an infinite set of modalities) for which the provability relation admits an axiomatization such that the set of instances of the infinitary proof rules (if any) is countable, then for any consistent formula $\varphi$, there exists a maximally-consistent set of formulas containing $\varphi$. Since we manage to replace CAR with an infinitary rule having a countable set of instances, we can apply this result and prove strong completeness for each of the three logics.

The contribution of this paper consists in the novelty of the proof method for strong completeness. We have used already these types of techniques in [13], where we proved a Stone duality for PMPs. That result implies strong completeness for the logic for PMPs but the logical aspects were not spelled out in that paper; there we concentrated on the algebraic versions of the logic and proved a duality theorem. In this paper we spell out the completeness theorem explicitly and, in addition, demonstrate that, in fact, the proof method can be used for the other two logics as well. Though these logics are superficially very similar, the axiomatizations are different and none of the completeness theorems follow directly from the others.

We also show that the infinitary axiom needed to replace CAR can be obtained by a lifting of the so-called archimedean axioms already present in the old versions of these axiomatizations. We are confident that similar results can be obtained for the gen- 
eral case of the measurable polynomial functors on the category of measurable spaces considered in [11], but we do not have such a result yet.

\section{Background}

Let $\mathbb{Q}_{0}=\mathbb{Q} \cap[0,1], \mathbb{Q}^{+}=\mathbb{Q} \cap[0, \infty), \mathbb{R}_{0}=\mathbb{R} \cap[0,1]$, and $\mathbb{R}^{+}=\mathbb{R} \cap[0, \infty)$.

\subsection{Measurable Spaces and Measures}

In this section we introduce a few concepts and results from measure theory that we will find useful. For more details, we refer the reader to $[3,8]$.

Let $M$ be an arbitrary nonempty set.

A field (of sets) over $M$ is a boolean algebra of subsets of $M$ under the usual settheoretic boolean operations. A $\sigma$-algebra (also called a $\sigma$-field) over $M$ is a field of sets over $M$ closed under countable union. The tuple $(M, \Sigma)$ where $\Sigma$ is a $\sigma$-algebra over $M$, is called a measurable space and the elements of $\Sigma$ measurable sets.

If $\Omega \subseteq 2^{M}$, the $\sigma$-algebra generated by $\Omega$, denoted $\sigma(\Omega)$, is the smallest $\sigma$-algebra containing $\Omega$. Every topological space has a natural $\sigma$-algebra associated with it, namely the one generated by the open sets. This is called the Borel algebra of the space, and the measurable sets are called Borel sets.

Given two measurable spaces $(M, \Sigma)$ and $(N, \Omega)$, a function $f: M \rightarrow N$ is measurable if $f^{-1}(T) \in \Sigma$ for all $T \in \Omega$. We use $\llbracket M \rightarrow N \rrbracket$ to denote the family of measurable functions from $(M, \Sigma)$ to $(N, \Omega)$.

A nonnegative real-valued set function $\mu$ is finitely additive if $\mu(A \cup B)=\mu(A)+\mu(B)$ whenever $A \cap B=\varnothing$. We say that $\mu$ is countably subadditive if $\mu\left(\bigcup_{i} A_{i}\right) \leq \sum_{i} \mu\left(A_{i}\right)$ for a countable family of measurable sets, and we say that $\mu$ is countably additive if $\mu\left(\cup_{i} A_{i}\right)=$ $\sum_{i} \mu\left(A_{i}\right)$ for a countable pairwise-disjoint family of measurable sets. Finite additivity implies monotonicity and countable additivity implies certain continuity properties; see the references for precise statements.

Given a measurable space $(M, \Sigma)$, a countably additive set function $\mu: \Sigma \rightarrow \mathbb{R}^{+}$is a measure on $(M, \Sigma)$. A measure $\mu: \Sigma \rightarrow \mathbb{R}_{0}$ is a subprobability measure. Thus, for a subprobability measure $\mu(M) \leq 1$; if in addition $\mu(M)=1, \mu$ is a probability measure. We use $\Delta(M, \Sigma), \Pi(M, \Sigma)$ and $\Pi^{*}(M, \Sigma)$ to denote the set of measures, probability measures and subprobability measures on $(M, \Sigma)$ respectively.

We view $\Delta(M, \Sigma)$ as a measurable space by defining the $\sigma$-algebra generated by the sets $\{\mu \in \Delta(M, \Sigma) \mid \mu(S) \geq r\}$ for $S \in \Sigma$ and $r \in \mathbb{R}^{+}$. This is the least $\sigma$-algebra on $\Delta(M, \Sigma)$ such that all maps $\mu \mapsto \mu(S): \Delta(M, \Sigma) \rightarrow \mathbb{R}^{+}$for $S \in \Sigma$ are measurable, where the set of positive reals is endowed with the $\sigma$-algebra generated by all rational intervals, i.e. the Borel $\sigma$-algebra. Similarly, $\Pi(M, \Sigma)$ and $\Pi^{*}(M, \Sigma)$ can be viewed as measurable spaces by defining the $\sigma$-algebras generated by the sets $\{\mu \in \Pi(M, \Sigma) \mid \mu(S) \geq r\}$ and $\left\{\mu \in \Pi^{*}(M, \Sigma) \mid \mu(S) \geq r\right\}$ respectively, defined for $S \in \Sigma$ and $r \in[0,1]$.

The next theorem is a key tool in our constructions.

Theorem 1. [Theorem 11.3 of [3]] Let $\mathcal{F} \subseteq 2^{M}$ be a field of sets. Let $\mu: \mathcal{F} \rightarrow \mathbb{R}^{+}$be finitely additive and countably subadditive. Then $\mu$ extends uniquely to a measure on $\sigma(\mathcal{F})$. 


\subsection{Analytic Spaces}

Recall that a topological space is said to be separable if it contains a countable dense subset and second countable if its topology has a countable base. Second countability implies separability, but not vice versa; however, the two concepts coincide for metric spaces. A Polish space is the topological space underlying a complete separable metric space. An analytic space is a continuous image of a Polish space in a Polish space.

Analytic spaces enjoy remarkable properties that were crucial in proving the logical characterization of bisimulation $[6,16]$. We note that the completeness theorems proved in $[4,15,21]$ were established for Markov processes defined on analytic spaces.

\subsection{The Baire Category Theorem}

The Baire category theorem is a topological result with important applications in logic. It is used to prove the Rasiowa-Sikorski lemma $[17,10]$ which is crucial for this paper.

A subset $D$ of a topological space $X$ is dense if its closure $\bar{D}$ is all of $X$. Equivalently, a dense set is one intersecting every nonempty open set. A set $N \subseteq X$ is nowhere dense if every nonempty open set contains a nonempty open subset disjoint from $N$. A set is of the first category or meager if it is a countable union of nowhere dense sets.

A Baire space is one in which the intersection of countably many dense open sets is dense. It follows from these definitions that the complement of a first category set is dense in any Baire space. Baire originally proved that the real line is a Baire space. More generally, every Polish space is Baire and every locally compact Hausdorff space is Baire. For us, the relevant version is the following special case: every compact Hausdorff space is Baire.

Definition 2. Let $\mathcal{B}$ be a boolean algebra and let $T \subseteq \mathcal{B}$ be such that $T$ has a greatest lower bound $\wedge T$ in $\mathcal{B}$. An ultrafilter (maximal filter) $U$ is said to respect $T$ if $T \subseteq U$ implies that $\wedge T \in U$. If $\mathcal{T}$ is a family of subsets of $\mathcal{B}$, we say that an ultrafilter $U$ respects $\mathcal{T}$ if it respects every member of $\mathcal{T}$.

Theorem 3 (Rasiowa-Sikorski lemma [17]). For any boolean algebra $\mathcal{B}$ and any countable family $\mathcal{T}$ of subsets of $\mathcal{B}$, each member of which has a meet in $\mathcal{B}$, and for any nonzero $x \in \mathcal{B}$, there exists an ultrafilter in $\mathcal{B}$ that contains $x$ and respects $\mathcal{T}$.

\section{Markov Processes}

In this section we introduce three classes of models of probabilistic systems with a continuous state space: (i) probabilistic Markov processes (PMPs), (ii) subprobabilistic Markov processes (SMPs) and (iii) general Markov processes (GMPs). The first two classes contain the systems for which the transition from a state to a measurable set of states is characterized by its probability. The third class represents the systems with continuous-time transitions, i.e., the probability of a transition from a state to a measurable set of states depends on time. In earlier papers, they were called labeled Markov processes to emphasize the fact that there were multiple possible actions, but here we will suppress the labels, as they do not contribute any relevant structure for our results. 
Definition 4 (Markov process). Given an analytic space $(M, \Sigma)$,

- a probabilistic Markov process is a measurable mapping $\theta \in \llbracket M \rightarrow \Pi(M, \Sigma) \rrbracket$;

- a subprobabilistic Markov process is a measurable mapping $\theta \in \llbracket M \rightarrow \Pi^{*}(M, \Sigma) \rrbracket$;

- a general Markov process is a measurable mapping $\theta \in \llbracket M \rightarrow \Delta(M, \Sigma) \rrbracket$.

In what follows we identify a Markov process with the tuple $\mathcal{M}=(M, \Sigma, \theta) ; M$ is called the support set, denoted by $\operatorname{supp}(\mathcal{M})$, and $\theta$ is called the transition function.

If $\mathcal{M}=(M, \Sigma, \theta)$ is a (probabilistic/subprobabilistic/general) Markov process, then for $m \in M, \theta(m)$ is a (probabilistic/subprobabilistic/general) measure on the state space $(M, \Sigma)$. If $\mathcal{M}$ is a PMP or a SMP, the value $\theta(m)(N)$ for $N \in \Sigma$ represents the probability of a transition from $m$ to a state in $N$; otherwise, if $\mathcal{M}$ is a GMP, then $\theta(m)$ is a measure on the state space and the value $\theta(m)(N) \in \mathbb{R}^{+}$represents the rate of an exponentially distributed random variable that characterizes the transition from $m$ to a state in $N$.

The condition that $\theta$ is measurable is equivalent to the condition that for fixed $N \in \Sigma$, the function $m \mapsto \theta(m)(N)$ is measurable (see e.g. Proposition 2.9 of [7]).

\section{Markovian Logics}

Markovian logics are multi-modal logics for semantics based on the three classes of Markov processes introduced in the previous section. They have been introduced and studied in various contexts $[1,2,14,12,19,9,4,15]$. In addition to the boolean operators, these logics are equipped with modal operators of type $L_{r}$ for rational numbers $r$ that are used to approximate the numerical labels of the transitions. Intuitively, the formula $L_{r} \varphi$ is satisfied by $m \in \mathcal{M}$ whenever the probability/rate of a transition from $m$ to a state satisfying the logical property $\varphi$ is at least $r$.

In this paper we study three Markovian logics: the probabilistic Markovian logic (PML), the subprobabilistic Markovian logic (SML) and the general Markovian logic (GML); they are interpreted on PMPs, SMPs and GMPs respectively. Despite their apparent similarities, we have found it necessary to treat these logics separately because of subtle technical differences that make a uniform treatment difficult.

\subsection{Syntax and Semantics}

Definition 5. Given a countable set $\mathcal{P}$ of atomic propositions, the grammars below define the sets of formulas $\mathcal{L}(\Pi)$ of probabilistic and subprobabilistic Markovian logic and $\mathcal{L}(\Delta)$ of general Markovian logic

$$
\begin{array}{lll}
\mathcal{L}(\Pi): & \varphi::=p|\neg \varphi| \varphi \wedge \varphi \mid L_{r} \varphi, & \text { for arbitrary } p \in \mathcal{P} \text { and } r \in \mathbb{Q}_{0} \\
\mathcal{L}(\Delta): & \varphi::=p|\neg \varphi| \varphi \wedge \varphi \mid L_{r} \varphi, & \text { for arbitrary } p \in \mathcal{P} \text { and } r \in \mathbb{Q}^{+}
\end{array}
$$

For each of these logics we assume that the usual boolean operators $T, \perp, \vee, \rightarrow$ are available as derived constructs as well as the additional derived operator

$$
L_{r_{1} \cdots r_{n}} \varphi=L_{r_{1}} \cdots L_{r_{n}} \varphi
$$

defined for $r_{1}, \ldots, r_{n} \in \mathbb{Q}_{0}$ for $\mathcal{L}(\Pi)$ and for $r_{1}, \ldots, r_{n} \in \mathbb{R}^{+}$for $\mathcal{L}(\Delta)$. 
To differentiate the probabilistic and the subprobabilistic logics, which have the same syntax but different semantics, we denote in what follows by $\mathcal{L}\left(\Pi^{*}\right)$ the logic interpreted on subprobabilistic distributions and we use $\mathcal{L}(\Pi)$ to refer to the logic interpreted on probabilistic distributions.

In what follows we define en masse the semantics for three logics using a generic $\mathcal{L}$ that ranges over the set $\left\{\mathcal{L}(\Pi), \mathcal{L}\left(\Pi^{*}\right), \mathcal{L}(\Delta)\right\}$. However, each of the following concepts has to be properly interpreted in each case. Let $\mathcal{M}=(M, \Sigma, \theta)$ be a PMP when we consider $\mathcal{L}=\mathcal{L}(\Pi)$, an SMP when we consider $\mathcal{L}=\mathcal{L}\left(\Pi^{*}\right)$ and an GMP when we consider $\mathcal{L}=\mathcal{L}(\Delta)$. Let $m \in M$ be an arbitrary state and $i: M \rightarrow 2^{\mathcal{P}}$ an arbitrary interpretation function for the atomic propositions. The semantics of the three logics is defined as follows.

- $\mathcal{M}, m, i \vDash p$ iff $p \in i(m)$,

- $\mathcal{M}, m, i \vDash \varphi \wedge \psi$ iff $\mathcal{M}, m, i \vDash \varphi$ and $\mathcal{M}, m, i \vDash \psi$,

- $\mathcal{M}, m, i \vDash \neg \varphi$ iff not $\mathcal{M}, m, i$ \& $\varphi$.

- $\mathcal{M}, m, i \vDash L_{r} \varphi$ iff $\theta(m)\left(\llbracket \varphi \rrbracket_{\mathcal{M}}^{i}\right) \geq r$, where $\llbracket \varphi \rrbracket_{\mathcal{M}}^{i}=\{m \in M \mid \mathcal{M}, m, i \vDash \varphi\}$.

For the last clause to make sense, $\llbracket \varphi \rrbracket_{\mathcal{M}}^{i}$ must be measurable. This is guaranteed, for each of the three types of Markov process, by the fact that $\theta$ is a measurable mapping between the measurable space of states and the measurable space of probabilistic/subprobabilistic/general distributions (see e.g. [4] for a complete proof).

Given $\mathcal{M}=(M, \Sigma, \theta)$ and $i$, we say that $m \in M$ satisfies $\varphi$ if $\mathcal{M}, m, i \vDash \varphi$. We write $\mathcal{M}, m, i \not \varphi$ if it is not the case that $\mathcal{M}, m, i \vDash \varphi$; and we write $\mathcal{M}, m, i \vDash \Phi$ if $\mathcal{M}, m, i$ ₹ $\varphi$ for all $\varphi \in \Phi$. We write $\Phi \vDash \varphi$ if $\mathcal{M}, m, i \vDash \varphi$ whenever $\mathcal{M}, m, i \vDash \Phi$. A formula or set of formulas is satisfiable if there exist an MP $\mathcal{M}$, an interpretation function $i$ for $\mathcal{M}$ and $m \in \operatorname{supp}(\mathcal{M})$ that satisfies it. We say that $\varphi$ is valid and write $\vDash \varphi$, if $\neg \varphi$ is not satisfiable.

In what follows, when we have to differentiate between the three semantics, we will use indexes: $k_{\Pi}$ will be used for PML, $\vDash_{\Pi^{*}}$ for SML and $k_{\Delta}$ for GML.

\subsection{Hilbert-style Axiomatizations}

We now present Hilbert-style axiomatic systems for the three logics. These axiomatic systems are meant to include the axioms of propositional logic; we do not write propositional axioms explicitly. In the next section we prove that these system are strongly complete for their semantics, meaning that an arbitrary formula $\varphi$ can be proven from an arbitrary set $\Phi$ of formulae if and only if the models of $\Phi$ are also models of $\varphi$.

As we did for the semantics, we introduce the concepts related to the provability en masse. However, they have a specific meaning for each logic and depend directly of each particular provability relation.

As usual, for an arbitrary formula $\varphi, \vdash \varphi$ denotes the fact that $\varphi$ is an axiom or a theorem in the system. If $\Phi$ is a set of formulas, we write $\Phi \vdash \varphi$ and say that $\Phi$ derives $\varphi$ if $\varphi$ is provable from the axioms and the extra assumptions $\Phi$; we implicitly assume that the provability relation is adapted for the infinitary proofs allowed by the axiomatic systems. A formula or set of formulas is consistent if it cannot derive $\perp$. We say that $\Phi$ is maximally consistent if it is consistent and it has no proper consistent extensions. 
When we have to differentiate between the three provability relations, we will use indexes: $\vdash_{\Pi}$ will be used for PML, $\vdash_{\Pi^{*}}$ for SML and $\vdash_{\Delta}$ for GML.

The axiomatic system of PML is listed in Table 1. The axioms and the rules are stated for arbitrary $\varphi, \psi \in \mathcal{L}$ and arbitrary $r, s, r_{1}, . ., r_{k} \in \mathbb{Q}_{0}$ for $k \geq 0$.

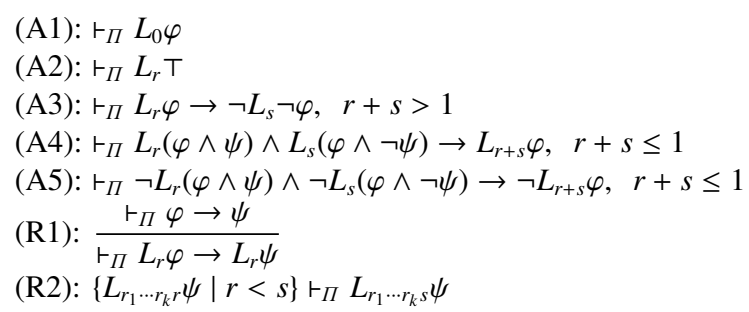

Table 1. The axioms of $\mathcal{L}(\Pi)$

A similar axiomatic system was studied in $[19,20]$. The novelty of our axiomatization is the rule (R2). In [19], for proving the strongly completeness of the axiomatic system, Lindenbaum's lemma is assumed as a meta-axiom and instead of (R2), the rules in Table 2 are used, stated for arbitrary $\varphi \in \mathcal{L}(\Pi)$ and arbitrary set $\Phi \subseteq \mathcal{L}(\Pi)$ closed under conjunction, where $L_{r} \Phi=\left\{L_{r} \psi \mid \psi \in \Phi\right\}$.

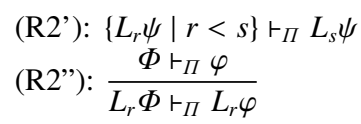

Table 2. Zhou's rules of $\mathcal{L}(\Pi)$

While (R2') is an instance of (R2), (R2") is much stronger and it has an uncountable set of instances. This makes the proof of the existence of the maximally consistent sets difficult. In our case that fact that (R2) has countably many instances means that the existence of maximally consistent sets is guaranteed by the Rasiowa-Sikorski lemma.

Before introducing the axiomatization of SML, notice that (A3) guarantees that the semantics must use distributions bounded by 1 while (A2) guarantees that these are probability distributions.

The axiomatic system of SML is listed in Table 3. The axioms and the rules are stated for arbitrary $\varphi, \psi \in \mathcal{L}$ and arbitrary $r, s, r_{1}, . ., r_{k} \in \mathbb{Q}_{0}$ for $k \geq 0$.

Notice the difference between this system and the previous one. For SML the axiom (A2) is not sound anymore, since for a subprobability distribution the measure of the entire space can be smaller than 1. However, (A2') which replaces (A2) is also sound for PML.

The axiomatic system of GML is listed in Table 4. The axioms and the rules are stated for arbitrary $\varphi, \psi \in \mathcal{L}$ and arbitrary $r, s, r_{1}, . ., r_{k} \in \mathbb{Q}^{+}$for $k \geq 0$. 


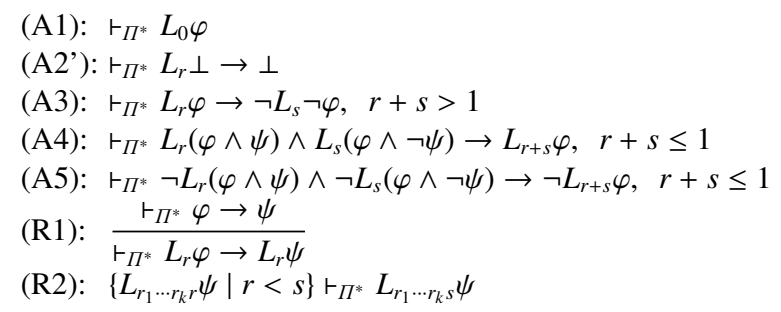

Table 3. The axioms of $\mathcal{L}\left(\Pi^{*}\right)$

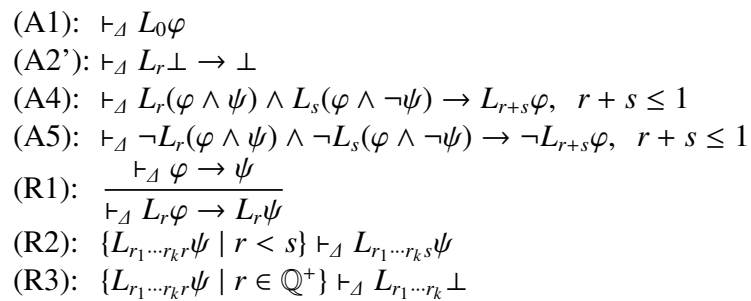

Table 4. The axioms of $\mathcal{L}(\Delta)$

The difference with respect to the axiomatic system of SML is that the axiom (A3) is not sound anymore. Moreover, the indexes of the modal operator can be any positive rational, meaning that these axioms have more instances than the corresponding ones in the other two systems. Since the semantics does not allow infinite measures, rule (R3) guarantees that divergent sequences of modalities prefixing some formula generates an inconsistent set of formulas.

Strong completeness for this logic was proven in [15] where, as in the case of Zhou's completeness for PML, Lindenbaum's lemma is postulated and the rules (R2') and (R2") are involved.

The next theorem states the soundness of the axioms of the three logics for their corresponding semantics

Theorem 6. [Soundness]

1. The axiomatization of PML is sound for the PMPs semantics, i.e.,

$$
\text { for any } \varphi \in \mathcal{L}(\Pi), \vdash_{\Pi} \varphi \text { implies } \vDash_{\Pi} \varphi \text {. }
$$

2. The axiomatization of SML is sound for the SMPs semantics, i.e.,

$$
\text { for any } \varphi \in \mathcal{L}\left(\Pi^{*}\right), \vdash_{\Pi^{*}} \varphi \text { implies } \vDash_{\Pi^{*}} \varphi \text {. }
$$

3. The axiomatization of GML is sound for the GMPs semantics, i.e.,

$$
\text { for any } \varphi \in \mathcal{L}(\Delta), \vdash_{\Delta} \varphi \text { implies } \vDash_{\Delta} \varphi \text {. }
$$




\subsection{Canonical Models}

In this section we construct canonical models for the three logics. The canonical model for a logic $\mathcal{L} \in\left\{\mathcal{L}(\Pi), \mathcal{L}\left(\Pi^{*}\right), \mathcal{L}(\Delta)\right\}$ is a Markov processes $\mathcal{M}_{\mathcal{L}}=\left(\mathcal{U}_{\mathcal{L}}, \Sigma_{\mathcal{L}}, \theta_{\mathcal{L}}\right)$ having the set $\mathcal{U}_{\mathcal{L}}$ of $\mathcal{L}$-maximally consistent sets of formulas as the state space and satisfying the property that for any $\varphi \in \mathcal{L}$ and $u \in \mathcal{U}_{\mathcal{L}}, \mathcal{M}_{\mathcal{L}}, u, i_{\mathcal{L}} \vDash \varphi$ iff $\varphi \in u$, where $i_{\mathcal{L}}$ is an appropriate interpretation function.

In order to complete such a construction, we have to:

- prove that $\mathcal{U}_{\mathcal{L}} \neq \varnothing$ for each $\mathcal{L} \in\left\{\mathcal{L}(\Pi), \mathcal{L}\left(\Pi^{*}\right), \mathcal{L}(\Delta)\right\}$

- define $\Sigma_{\mathcal{L}}$ such that $\left(\mathcal{U}_{\mathcal{L}}, \Sigma_{\mathcal{L}}\right)$ is an analytic space;

- define a measure $\theta_{\mathcal{L}}$ on $\left(\mathcal{U}_{\mathcal{L}}, \Sigma_{\mathcal{L}}\right)$;

- define an interpretation function $i_{\mathcal{L}}$ such that for any $\varphi \in \mathcal{L}, \llbracket \varphi \rrbracket_{\mathcal{M}_{\mathcal{L}}}^{i_{\mathcal{L}} \in \Sigma_{\mathcal{L}}}$;

- and prove the Truth Lemma stating that $\mathcal{M}_{\mathcal{L}}, u, i_{\mathcal{L}} \vDash \varphi$ iff $\varphi \in u$.

Lemma 7. For $\mathcal{L} \in\left\{\mathcal{L}(\Pi), \mathcal{L}\left(\Pi^{*}\right), \mathcal{L}(\Delta)\right\}$ with the proof systems previously defined, the set $\mathcal{U}_{\mathcal{L}}$ of $\mathcal{L}$-maximally consistent sets is nonempty.

Proof. Note that in each case $\mathcal{L}$ forms a boolean algebra and the instances of all the axioms and rules define a countable family of subsets of $\mathcal{L}$, each member of which has the meet in $\mathcal{L}$; in particular the instances of (R2) define the subsets $\left\{L_{r_{1} \cdots r_{k} r} \psi \mid r<s\right\}$ of $\mathcal{L}$ each having the meet $L_{r_{1} \ldots r_{k} s} \psi \in \mathcal{L}$. Observe also that a set $u \subseteq \mathcal{L}$ is a $\mathcal{L}$-maximally consistent set iff it is a boolean ultrafilter that respects all the instances of the axioms of $\mathcal{L}$. Consequently, the Rasiowa-Sikorski Lemma guarantees that $\mathcal{U}_{\mathcal{L}} \neq \varnothing$.

Let $(\mathcal{L})=\{(\varphi) \mid \varphi \in \mathcal{L}\}$, where $(\varphi)=\left\{u \in \mathcal{U}_{\mathcal{L}} \mid \varphi \in u\right\}$. Using this, we define $\Sigma_{\mathcal{L}}=$ $\sigma((\mathcal{L}))$. The space $\left(\mathcal{U}_{\mathcal{L}}, \Sigma_{\mathcal{L}}\right)$ is an analytic space for each $\mathcal{L} \in\left\{\mathcal{L}(\Pi), \mathcal{L}\left(\Pi^{*}\right), \mathcal{L}(\Delta)\right\}$. The proof for the probabilistic case — which is decidedly non-trivial — can be found in [13] and works similarly for the other two cases.

The next step in our construction is to define an appropriate measure $\theta_{\mathcal{L}}$ on $\left(\mathcal{U}_{\mathcal{L}}, \Sigma_{\mathcal{L}}\right)$. To do this we prove the following lemma.

Lemma 8. 1. For arbitrary $u \in \mathcal{U}_{\mathcal{L}(\Pi)}$ and $\varphi \in \mathcal{L}(\Pi)$, or $u \in \mathcal{U}_{\mathcal{L}\left(\Pi^{*}\right)}$ and $\varphi \in \mathcal{L}\left(\Pi^{*}\right)$,

$$
x_{u}^{\varphi}=\sup \left\{r \in \mathbb{Q}_{0} \mid L_{r} \varphi \in u\right\}=\inf \left\{r \in \mathbb{Q}_{0} \mid \neg L_{r} \varphi \in u\right\} .
$$

Moreover, if $x_{u}^{\varphi} \in \mathbb{Q}$, then $L_{x_{u}^{\varphi}} \varphi \in u$.

2. For arbitrary $u \in \mathcal{U}_{\mathcal{L}(\Delta)}$ and $\varphi \in \mathcal{L}(\Delta)$,

$$
x_{u}^{\varphi}=\sup \left\{r \in \mathbb{Q}^{+} \mid L_{r} \varphi \in u\right\}=\inf \left\{r \in \mathbb{Q}^{+} \mid \neg L_{r} \varphi \in u\right\} \in \mathbb{R}^{+} .
$$

Moreover, if $x_{u}^{\varphi} \in \mathbb{Q}$, then $L_{x_{u}^{\varphi}} \varphi \in u$.

The previous lemma allows us to define, for each $\mathcal{L} \in\left\{\mathcal{L}(\Pi), \mathcal{L}\left(\Pi^{*}\right), \mathcal{L}(\Delta)\right\}$ and arbitrary $u \in \mathcal{U}_{\mathcal{L}}, \varphi \in \mathcal{L}$,

$$
\left.\theta_{\mathcal{L}}(u)(|\varphi|)\right)=\sup \left\{r \in \mathbb{Q}^{+} \mid L_{r} \varphi \in u\right\} .
$$

Obviously, $\theta_{\mathcal{L}}(u)$ is a set function defined on the field $(\mathcal{L})$ and Theorem 1 ensures us that it can be uniquely extended to a measure on $\Sigma_{\mathcal{L}}$ if it is finitely additive and countable subadditive on $(\mathcal{L})$. This is what we prove next. 
Lemma 9. For all $u \in \mathcal{U}_{\mathcal{L}}$, the function $\theta_{\mathcal{L}}(u)$ is finitely additive.

Now we prove that the function is also countable subadditive and this is a central result of the paper where we make use of (R2). In related papers, to prove a similar result a so-called countable additivity axiom was used. This is an infinitary axiom with uncountable instances $[11,21,15]$.

The main technical lemma that lies at the heart of the construction is proved in our previous paper on Stone duality [13] for the probabilistic case. The proof can be similarly done for the other two cases.

Lemma 10. For $u \in \mathcal{U}_{\mathcal{L}}$, the function $\theta_{\mathcal{L}}(u)$ is countably subadditive.

The previous lemmas guarantees that $\theta_{\mathcal{L}}$ can be extended to a measure on $\left(\mathcal{U}_{\mathcal{L}}, \Sigma_{\mathcal{L}}\right)$. From the construction we also obtain that $\theta_{\mathcal{L}(\Pi)}$ is a probabilistic measure and $\theta_{\mathcal{L}\left(\Pi^{*}\right)}$ is a subprobabilistic measure.

\section{Theorem 11 (Canonical models).}

1. $\mathcal{M}_{\mathcal{L}(I)}=\left(\mathcal{U}_{\mathcal{L}(I)}, \Sigma_{\mathcal{L}(I)}\right)$ is a probabilistic Markov process;

2. $\mathcal{M}_{\mathcal{L}\left(\Pi^{*}\right)}=\left(\mathcal{U}_{\mathcal{L}\left(\Pi^{*}\right)}, \Sigma_{\mathcal{L}\left(\Pi^{*}\right)}\right)$ is a subprobabilistic Markov process;

3. $\mathcal{M}_{\mathcal{L}(\Delta)}=\left(\mathcal{U}_{\mathcal{L}(\Delta)}, \Sigma_{\mathcal{L}(\Delta)}\right)$ is a general Markov process.

Proof. In the generic case we only need to verify that $\theta_{\mathcal{L}}$ is a measurable function. Let $\varphi \in \mathcal{L}$, and $r \in[0,1]$ for $\mathcal{L}(\Pi)$ and $\mathcal{L}\left(\Pi^{*}\right)$ and $r \in \mathbb{R}^{+}$for $\mathcal{L}(\Delta)$. Consider $\left(r_{i}\right)_{i} \subseteq \mathbb{Q}$ an increasing sequence with supremum $r$. Let $X=\left\{\mu \in \Pi\left(\mathcal{U}_{\mathcal{L}(\Pi)}, \Sigma_{\mathcal{L}(\Pi)}\right) \mid \mu((\varphi \varphi)) \geq\right.$ $r\}$ for $\mathcal{L}(\Pi), X=\left\{\mu \in \Pi^{*}\left(\mathcal{U}_{\mathcal{L}\left(\Pi^{*}\right)}, \Sigma_{\mathcal{L}\left(\Pi^{*}\right)}\right) \mid \mu((\varphi)) \geq r\right\}$ for $\mathcal{L}\left(\Pi^{*}\right)$ and $X=\{\mu \in$ $\left.\Delta\left(\mathcal{U}_{\mathcal{L}(\Delta)}, \Sigma_{\mathcal{L}(\Delta)}\right) \mid \mu(|\varphi|) \geq r\right\}$ for $\mathcal{L}(\Delta)$. It suffices to prove, in each case, that $\theta_{\mathcal{L}}^{-1}(X) \in$ $\Sigma_{\mathcal{L}}$. But

$$
\theta_{\mathcal{L}}^{-1}(X)=\left\{u \in \mathcal{U}_{\mathcal{L}} \mid \theta(u)(|\varphi|) \geq r\right\}=\bigcap_{i}\left\{u \in \mathcal{U}_{\mathcal{L}} \mid \theta_{\mathcal{L}}(u)(|\varphi|) \geq r_{i}\right\}=\bigcap_{i}\left|L_{r_{i}} \varphi\right| \in \Sigma_{\mathcal{L}} .
$$

We define an interpretation function $i_{\mathcal{L}}$ for arbitrary $u \in \mathcal{U}_{\mathcal{L}}$ by $i_{\mathcal{L}}(u)=u \cap \mathcal{P}$. Now we are ready to prove the Truth Lemma.

Lemma 12 (Truth Lemma). For $\mathcal{L} \in\left\{\mathcal{L}(\Pi), \mathcal{L}\left(\Pi^{*}\right), \mathcal{L}(\Delta)\right\}, \Phi \subseteq \mathcal{L}$ and $u \in \mathcal{U}_{\mathcal{L}}$,

$$
\mathcal{M}_{\mathcal{L}}, u, i_{\mathcal{L}} \vDash \Phi \text { iff } \Phi \subseteq u \text {. }
$$

Proof. It is sufficient to prove inductively that for any $\varphi \in \mathcal{L}, \mathcal{M}_{\mathcal{L}}, u, i_{\mathcal{L}} \vDash \varphi$ iff $\varphi \in u$. The case $\varphi \in \mathcal{P}$ and the boolean cases are trivial.

The case $\varphi=L_{r} \psi:(\Longrightarrow)$ Suppose that $\mathcal{M}_{\mathcal{L}}, u, i_{\mathcal{L}} \vDash \varphi$ and $\varphi \notin u$. Hence $\neg \varphi \in u$. Let $x_{u}^{\varphi}=\inf \left\{r \in \mathbb{Q} \mid \neg L_{r} \psi \in u\right\}$. Then, from $\neg L_{r} \psi \in u$, we obtain $r \geq x_{u}^{\varphi}$. But $\mathcal{M}_{\mathcal{L}}, u, i_{\mathcal{L}} \vDash L_{r} \psi$ is equivalent with $\left.\theta_{\mathcal{L}}(u)(|\psi|)\right) \geq r$, i.e. $x_{u}^{\varphi} \geq r$. Hence, $x_{u}^{\varphi}=r \in \mathbb{Q}$ and Lemma 8 implies $L_{x_{u}^{\psi}} \varphi \in u$, i.e., $\varphi \in u$ - contradiction.

$(\Longleftarrow)$ If $L_{r} \psi \in u$, then $r \leq x_{u}^{\varphi}$, i.e., $\left.r \leq \theta_{\mathcal{L}}(u)(|\psi|)\right)$. Hence, $\mathcal{M}_{\mathcal{L}}, u, i_{\mathcal{L}} \vDash L_{r} \psi$.

The Truth Lemma allows us to prove strong completeness for all three logics. 
Theorem 13 (Completeness). For $\mathcal{L} \in\left\{\mathcal{L}(\Pi), \mathcal{L}\left(\Pi^{*}\right), \mathcal{L}(\Delta)\right\}, \Phi \subseteq \mathcal{L}$ and $\varphi \in \mathcal{L}$

$$
\Phi \vDash \varphi \text { iff } \Phi \vdash \varphi .
$$

Proof. $(\Longleftarrow)$ This is a consequence of soundness, Theorem 6 .

$(\Longrightarrow)$ If $\Phi$ is inconsistent, the statement is trivially true. Suppose that $\Phi$ is consistent, and let $u \in \mathcal{U}_{\mathcal{L}}$ be an arbitrary maximally consistent set. We have that $\Phi \subseteq u$ iff $\mathcal{U}_{\mathcal{L}}, u, i_{\mathcal{L}} \vDash \Phi$ (from Truth Lemma). But if $\mathcal{U}_{\mathcal{L}}, u, i_{\mathcal{L}} \vDash \Phi$, since $\Phi \vDash \varphi$, we obtain that $\mathcal{U}_{\mathcal{L}}, u, i_{\mathcal{L}} \vDash \varphi$. Applying again the truth lemma we get $\varphi \in u$. Consequently, for an arbitrary maximally-consistent set $u \in \mathcal{U}_{\mathcal{L}}, \Phi \subseteq u$ implies $\varphi \in u$. Hence, $\Phi \vdash \varphi$.

\section{Conclusions and Related Work}

The most closely related work to ours is the work of Goldblatt [10] on the role of the Baire category theorem in completeness proofs, and his work on deduction systems for coalgebras [11]. The main difference between his work and ours is that we have replaced the Countable additivity Rule (CAR) that he uses, with a different infinitary axiom that has only countably many instances. Goldblatt uses CAR in order to show countable additivity of the measures that he defines; this is where we have been able to use of the Rasiowa-Sikorski lemma. As far as we know this is a new idea. Furthermore, Goldblatt's results are contingent on the assumption that consistent sets can be expanded to maximally consistent sets; we have essentially proved this fact for our logics.

Regarding the completeness proofs for Markovian logics, the results for probabilistic case were proved by Zhou in [19] and for the general case by Mardare-CardelliLarsen in [15]. In these papers the strong completeness is solved using CAR.

In this paper, we have used some of the results of our earlier paper [13] to show that we can obtain strong completeness theorems for three types of Markov processes and their related logics. The main technical lemmas about measure theory are in [13] but the canonical model constructions which use those facts are in the present paper. That paper focussed on algebra and duality whereas the present paper is primarily about logic and can be read independently.

A very tempting future research project is to extend these completeness theorems to the entire class of systems described as coalgebras of polynomial functors described by Goldblatt [11]. It is possible that the results of Pattinson and Schröder [18] will be useful for this.

Though the focus of the present paper has been on probabilistic systems and Markovian logics, the techniques may well apply to any non-compact modal logic. We are investigating whether there is a general way of introducing an infinitary axiom that will allow us to mimic the techniques of the present paper.

\section{Acknowledgments}

We would like to thank Ernst-Erich Doberkat, Rob Goldblatt, Jean Goubault-Larrecq, Larry Moss and Chunlai Zhou for helpful discussions.

Panangaden's research was supported by an NSERC grant. Mardare's research was supported by VKR Center of Excellence MT-LAB and by the Sino-Danish Basic Research Center IDEA4CPS. 


\section{References}

1. Robert Aumann. Interactive epistemology I: knowledge. International Journal of Game Theory, 28:263-300, 1999.

2. Robert Aumann. Interactive epistemology II: probability. International Journal of Game Theory, 28:301-314, 1999.

3. P. Billingsley. Probability and Measure. Wiley-Interscience, 1995.

4. Luca Cardelli, Kim G. Larsen, and Radu Mardare. Continuous markovian logic - from complete axiomatization to the metric space of formulas. In CSL, pages 144-158, 2011.

5. J. Desharnais, A. Edalat, and P. Panangaden. A logical characterization of bisimulation for labelled Markov processes. In proceedings of the 13th IEEE Symposium On Logic In Computer Science, Indianapolis, pages 478-489. IEEE Press, June 1998.

6. J. Desharnais, A. Edalat, and P. Panangaden. Bisimulation for labeled Markov processes. Information and Computation, 179(2):163-193, Dec 2002.

7. E.-E. Doberkat. Stochastic Relations. Foundations for Markov Transition Systems. Chapman and Hall, New York, 2007.

8. R. M. Dudley. Real Analysis and Probability. Wadsworth and Brookes/Cole, 1989.

9. R. Fagin and J. Y. Halpern. Reasoning about knowledge and probability. Journal of the ACM, 41(2):340-367, 1994.

10. R. Goldblatt. On the role of the Baire category theorem in the foundations of logic. Journal of Symbolic logic, pages 412-422, 1985.

11. R. Goldblatt. Deduction systems for coalgebras over measurable spaces. Journal of Logic and Computation, 20(5):1069-1100, 2010.

12. Aviad Heifetz and Philippe Mongin. Probability logic for type spaces. Games and Economic Behavior, 35(1-2):31-53, April 2001.

13. D. Kozen, K. G. Larsen, R. Mardare, and P. Panangaden. Stone duality for markov processes. In Proceedings of the 28th Annual IEEE Symposium on Logic in Computer Science: LICS 2013. IEEE Computer Society, 2013.

14. K. G. Larsen and A. Skou. Bisimulation through probablistic testing. Information and Computation, 94:1-28, 1991.

15. Radu Mardare, Luca Cardelli, and Kim G. Larsen. Continuous markovian logics - axiomatization and quantified metatheory. Logical Methods in Computer Science, 8(4), 2012.

16. Prakash Panangaden. Labelled Markov Processes. Imperial College Press, 2009.

17. H. Rasiowa and R. Sikorski. A proof of the completeness theorem of gödel. Fund. Math, 37:193-200, 1950.

18. Lutz Schröder and Dirk Pattinson. Modular algorithms for heterogeneous modal logics. In L. Arge, C. Cachin, T. Jurdzinski, and A. Tarlecki, editors, 34th Int. Colloq. Automata, Languages and Programming (ICALP 2007), volume 4596 of Lecture Notes in Computer Science, pages 459-471. Springer, 2007.

19. C. Zhou. A complete deductive system for probability logic with application to Harsanyi type spaces. $\mathrm{PhD}$ thesis, Indiana University, 2007.

20. Chunlai Zhou. Probability logic of finitely additive beliefs. J. Logic, Language and Information, 19(3):247-282, 2010.

21. Chunlai Zhou and Mingsheng Ying. Approximating Markov processes through filtration. Theoretical Computer Science, 446(0):75-97, 2012. 


\section{Appendix}

Proof (Proof of Lemma 8). 1. This proof can be found in [13], Lemma 17.

2. This result can be proven similarly to the previous case. However, to show that $x_{u}^{\varphi} \in$ $\mathbb{R}^{+}$, we use (R3) that guarantees the existence of a finite upper bound for $\left\{r \in \mathbb{Q}^{+} \mid\right.$ $\left.L_{r} \varphi \in u\right\}$.

Proof (Proof of Lemma 9). Suppose $\varphi, \psi \in \mathcal{L}$ and $(\varphi) \cap(\psi)=\varnothing$. Then $\vdash \varphi \wedge \psi \rightarrow \perp$. We wish to show that

$$
\left.\left.\theta_{\mathcal{L}}(u)(|\varphi \vee \psi|)=\theta_{\mathcal{L}}(u)(\mid \varphi \varphi)\right)+\theta_{\mathcal{L}}(u)(|\psi|)\right) .
$$

It suffices to show the inequality in both directions. For $\leq$, by the definition of $\theta$, it suffices to show

$$
\begin{aligned}
& \sup \left\{t \mid L_{t}(\varphi \vee \psi) \in u\right\} \\
& \leq \inf \left\{r \mid \neg L_{r} \varphi \in u\right\}+\inf \left\{s \mid \neg L_{s} \psi \in u\right\} \\
& =\inf \left\{r+s \mid \neg L_{r} \varphi \in u \text { and } \neg L_{s} \psi \in u\right\} \\
& =\inf \left\{r+s \mid \neg L_{r} \varphi \wedge \neg L_{s} \psi \in u\right\} ;
\end{aligned}
$$

that is, if $L_{t}(\varphi \vee \psi) \in u$ and $\neg L_{r} \varphi \wedge \neg L_{s} \psi \in u$, then $t \leq r+s$. But

$$
\begin{array}{r}
\vdash \neg L_{r} \varphi \wedge \neg L_{s} \psi \leftrightarrow \neg L_{r}((\varphi \vee \psi) \wedge \varphi) \wedge \neg L_{s}((\varphi \vee \psi) \wedge \neg \varphi) \text { and } \\
\vdash \neg L_{r}((\varphi \vee \psi) \wedge \varphi) \wedge \neg L_{s}((\varphi \vee \psi) \wedge \neg \varphi) \rightarrow \neg L_{r+s}(\varphi \vee \psi) \quad \text { by (A5), }
\end{array}
$$

thus $\neg L_{r+s}(\varphi \vee \psi) \in u$, and necessarily $t \leq r+s$.

The inequality in the opposite direction is similar, using (A4). We need to show

$$
\inf \left\{t \mid \neg L_{t}(\varphi \vee \psi) \in u\right\} \geq \sup \left\{r+s \mid L_{r} \varphi \wedge L_{s} \psi \in u\right\} ;
$$

that is, if $\neg L_{t}(\varphi \vee \psi) \in u$ and $L_{r} \varphi \wedge L_{s} \psi \in u$, then $t \geq r+s$. But

$$
\begin{array}{r}
\vdash L_{r} \varphi \wedge L_{s} \psi \leftrightarrow L_{r}((\varphi \vee \psi) \wedge \varphi) \wedge L_{s}((\varphi \vee \psi) \wedge \neg \varphi) \text { and } \\
\vdash L_{r}((\varphi \vee \psi) \wedge \varphi) \wedge L_{s}((\varphi \vee \psi) \wedge \neg \varphi) \rightarrow L_{r+s}(\varphi \vee \psi) \quad \text { by (A4), }
\end{array}
$$

thus $L_{r+s}(\varphi \vee \psi) \in u$ implying $r+s \leq t$.

Proof (Proof of Lemma 10). To prove that $\theta_{\mathcal{L}}$ is countably subadditive, it is sufficient to prove that it is continuous from above at $\varnothing$, i.e., that if $u \in \mathcal{U}_{\mathcal{L}}$ and $\left.\left(\psi_{0}\right\rangle\right) \geq\left|\psi_{1}\right| \geq \cdots$ with $\left.\left.\bigcap_{i}\right\rangle \psi_{i}\right\rangle=\varnothing$, then

$$
\inf _{i} \theta_{\mathcal{L}}(u)\left(\left(0 \psi_{i}\right)\right)=0
$$

1. We prove this firstly for $\mathcal{L}(\Phi)$ and $\mathcal{L}\left(\Pi^{*}\right)$.

Consider the countable set $\mathcal{F}$ of elements of the form $\alpha^{r}=L_{t_{1} \cdots t_{n} r} \varphi$ for $\varphi \in \mathcal{L}$ and rational $t_{1}, \ldots, t_{n}, r \geq 0$, parameterized by $r$. If $r<s$, then $\vdash \alpha^{s} \rightarrow \alpha^{r}$. Using (A4),

$$
\theta_{\mathcal{L}}(u)\left(\left(\left|\alpha^{r} \wedge \neg \alpha^{s}\right|\right) \leq \theta_{\mathcal{L}}(u)\left(\left(\alpha^{r}\right\rangle\right)-\theta(u)\left(\left(\alpha^{s} \mid\right) .\right.\right.
$$


Since $L_{t} \alpha^{r} \in u$ for all $r<s$ iff $L_{t} \alpha^{s} \in u$, therefore

$$
\theta_{\mathcal{L}}(u)\left(\left(\alpha^{s}\right\rangle\right)=\inf _{r<s} \theta_{\mathcal{L}}(u)\left(\left(\alpha^{r}\right\rangle\right) .
$$

Let $\varepsilon>0$ be an arbitrarily small positive number. For each $\alpha \in \mathcal{F}$ and $s \in \mathbb{Q}_{0}$, choose $\varepsilon_{\alpha}^{s}>0$ such that $\sum_{\alpha \in \mathcal{F}} \sum_{s \in \mathbb{Q}_{0}} \varepsilon_{\alpha}^{s}=\varepsilon$. By (1) and (2), we can choose $r_{\alpha}^{s}<s$ such that

$$
\theta_{\mathcal{L}}(u)\left(\left(\left|\alpha^{r_{\alpha}^{s}} \wedge \neg \alpha^{s}\right\rangle\right) \leq \theta_{\mathcal{L}}(u)\left(\left(\alpha^{r_{\alpha}^{s}}\right\rangle\right)-\theta_{\mathcal{L}}(u)\left(\left(\left|\alpha^{s}\right\rangle\right) \leq \varepsilon_{\alpha}^{s} .\right.\right.
$$

We call a set of formulas of $\mathcal{L}$ finitely-consistent if each finite subset of it is consistent. For an arbitrary $\psi \in \mathcal{L}$, let $(\psi)^{*}$ be the set maximally-finitely-consistent sets of formulas that contain $\psi$.

The assumption $\bigcap_{i}\left|\psi_{i}\right|=\varnothing$ implies that $\bigcap_{i}\left(\mid \psi_{i}\right)^{*}$ contains no maximally-consistent set, but only maximally-finitely-consistent sets that are inconsistent (due to (R2)). We have

$$
\left.\mathcal{U}_{\mathcal{L}}=\bigcap_{\alpha \in \mathcal{F}} \bigcap_{s \in \mathbb{Q}_{0}}\left(\bigcup_{r<s} \mid \neg \alpha^{r}\right)^{*} \cup\left(\alpha^{s}\right)^{*}\right) .
$$

Thus $\bigcap_{i}\left(\psi_{i}\right)=\varnothing$ is equivalent to the condition

$$
\left(\bigcap_{\alpha \in \mathcal{F}} \bigcap_{s \in \mathbb{Q}_{0}}\left(\bigcup_{r<s}\left(\neg \neg \alpha^{r}\right)^{*} \cup\left(\alpha^{s}\right)^{*}\right)\right) \cap \bigcap_{i}\left(\psi_{i}\right)^{*}=\varnothing .
$$

From this it follows that

$$
\left.\left(\bigcap_{\alpha \in \mathcal{F}} \bigcap_{s \in \mathbb{Q}_{0}}\left(\mid \neg \alpha^{r_{\alpha}^{s}}\right)^{*} \cup\left(\alpha^{s}\right)^{*}\right)\right) \cap \bigcap_{i}\left(\mid \psi_{i}\right)^{*}=\varnothing .
$$

The set of maximally-finitely-consistent sets form a Stone space (the maximallyfinitely-consistent sets are the ultarfilters of the support boolean algebra), which is a compact space and $(\psi)^{*}$ is a clopen for any $\psi \in \mathcal{L}$, see [13]. Consequently, there exist finite sets $C_{0} \subseteq \mathcal{F}$ and $S_{0} \subseteq \mathbb{Q} \cap[0,1]$ and $j \in \mathbb{N}$ such that

$$
\bigcap_{\alpha \in C_{0}} \bigcap_{s \in S_{0}}\left(\neg \alpha^{r_{\alpha}^{s}} \vee \alpha^{s}\right)^{*} \cap\left(\psi_{j}\right)^{*}=\varnothing,
$$

or in other words,

$$
\left.\left(\mid \psi_{j}\right)^{*} \subseteq \bigcup_{\alpha \in C_{0}} \bigcup_{s \in S_{0}}\left(\alpha^{r_{\alpha}^{s}} \wedge \neg \alpha^{s}\right)^{*}=0 \bigvee_{\alpha \in C_{0}} \bigvee_{s \in S_{0}}\left(\alpha^{r_{\alpha}^{s}} \wedge \neg \alpha^{s}\right)\right)^{*}
$$

This proves that,

$$
\vdash \psi_{j} \rightarrow \bigvee_{\alpha \in C_{0}} \bigvee_{s \in S_{0}}\left(\alpha^{r_{\alpha}^{s}} \wedge \neg \alpha^{s}\right)
$$


Consequently,

$$
\begin{aligned}
\theta_{\mathcal{L}}(u)\left(\left(\psi_{j}\right)\right) & \leq \theta_{\mathcal{L}}(u)\left(0 \bigvee_{\alpha \in C_{0}} \bigvee_{s \in S_{0}}\left(\alpha^{r_{\alpha}^{s}} \wedge \neg \alpha^{s}\right) \emptyset\right) \leq \sum_{\alpha \in C_{0}} \sum_{s \in S_{0}} \theta_{\mathcal{L}}(u)\left(\left(\alpha^{r_{\alpha}^{s}} \wedge \neg \alpha^{s}\right)\right) \\
& \leq \sum_{\alpha \in C_{0}} \sum_{s \in S_{0}} \varepsilon_{\alpha}^{s} \leq \varepsilon .
\end{aligned}
$$

As $\varepsilon>0$ was arbitrary, $\inf _{i} \theta_{\mathcal{L}}(u)\left(\left(\psi_{i}\right)\right)=0$.

2. To prove it for $\mathcal{L}(\Delta)$ we follow the same strategy only that we need to also consider the instances of (R3). We use the same notation.

As for the other case, we consider the countable set $\mathcal{F}$ of elements of the form $\alpha^{r}=L_{t_{1} \cdots t_{n} r} \varphi$ for $\varphi \in \mathcal{L}$ and rational $t_{1}, \ldots, t_{n}, r \geq 0$, parameterized by $r$ only that, in addition we also add the elements $\alpha^{\infty}=L_{t_{1} \cdots t_{n}} \perp$. As before, if $r<s$, then $\vdash \alpha^{s} \rightarrow \alpha^{r}$ and the inequality 1 holds. Similarly, the inequality 2 holds and, in addition, we also have

$$
\theta_{\mathcal{L}}(u)\left(\left(\mid \alpha^{\infty}\right)\right)=\inf _{r \in \mathbb{Q}^{+}} \theta_{\mathcal{L}}(u)\left(\left(\alpha^{r}\right)\right) .
$$

Let $\varepsilon>0$ be an arbitrarily small positive number. For each $\alpha \in \mathcal{F}$ and $s \in \mathbb{Q}^{+} \cup\{\infty\}$, choose $\varepsilon_{\alpha}^{s}>0$ such that $\sum_{\alpha \in \mathcal{F}} \sum_{s \in \mathbb{Q}^{+} \cup\{\infty\}} \varepsilon_{\alpha}^{s}=\varepsilon$. As before, we can choose $r_{\alpha}^{s}<s$ such that

$$
\theta_{\mathcal{L}}(u)\left(\left(\left|\alpha^{r_{\alpha}^{s}} \wedge \neg \alpha^{s}\right\rangle\right) \leq \theta_{\mathcal{L}}(u)\left(\left(\alpha^{r_{\alpha}^{s}}\right)\right)-\theta_{\mathcal{L}}(u)\left(\left(\alpha^{s}\right)\right) \leq \varepsilon_{\alpha}^{s} .\right.
$$

and $r_{\alpha}^{\infty} \in \mathbb{Q}^{+}$big enough such that

$$
\theta_{\mathcal{L}}(u)\left(\left(\alpha^{r_{\alpha}^{\infty}} \wedge \neg \alpha^{\infty}\right\rangle\right) \leq \theta_{\mathcal{L}}(u)\left(\left(\mid \alpha^{r_{\alpha}^{\infty}}\right)\right)-\theta_{\mathcal{L}}(u)\left(\left(\alpha^{\infty}\right\rangle\right) \leq \varepsilon_{\alpha}^{\infty} .
$$

This time we have

$$
\left.\mathcal{U}_{\mathcal{L}}=\bigcap_{\alpha \in \mathcal{F}} \bigcap_{s \in \mathbb{Q}^{+} \cup\{\infty\}}\left(\bigcup_{r<s} \mid \neg \alpha^{r}\right)^{*} \cup\left(\mid \alpha^{s}\right)^{*}\right) .
$$

and following the same rationament as in the previous case we obtain that there exist finite sets $C_{0} \subseteq \mathcal{F}$ and $S_{0} \subseteq \mathbb{Q}^{+} \cup\{\infty\}$ and $j \in \mathbb{N}$ such that

$$
\bigcap_{\alpha \in C_{0}} \bigcap_{s \in S_{0}}\left(\neg \alpha^{r_{\alpha}^{s}} \vee \alpha^{s}\right)^{*} \cap\left(\psi_{j}\right)^{*}=\varnothing,
$$

From here we obtain the same conclusion as for case 1, which is

$$
\vdash \psi_{j} \rightarrow \bigvee_{\alpha \in C_{0}} \bigvee_{s \in S_{0}}\left(\alpha^{r_{\alpha}^{s}} \wedge \neg \alpha^{s}\right)
$$

implying further

$$
\begin{aligned}
\theta_{\mathcal{L}}(u)\left(\left(\psi_{j}\right)\right) & \leq \theta_{\mathcal{L}}(u)\left(\left(\bigvee_{\alpha \in C_{0}} \bigvee_{s \in S_{0}}\left(\alpha^{r_{\alpha}^{s}} \wedge \neg \alpha^{s}\right)\right)\right) \leq \sum_{\alpha \in C_{0}} \sum_{s \in S_{0}} \theta_{\mathcal{L}}(u)\left(\left(\alpha^{r_{\alpha}^{s}} \wedge \neg \alpha^{s}\right)\right) \\
& \leq \sum_{\alpha \in C_{0}} \sum_{s \in S_{0}} \varepsilon_{\alpha}^{s} \leq \varepsilon
\end{aligned}
$$

